\section{GREGORY W. RUTECKI, MD, Section Editor}

Sarah A. Goldstein, MD

Adult Congenital Heart Disease Fellow,

Division of Cardiology, Duke University

Medical Center, Durham, NC
Lisa Sorenson, CNP

Department of Cardiovascular Medicine,

Cleveland Clinic, Cleveland, $\mathrm{OH}$
Jeffrey B. Chapa, MD

Section of Maternal-Fetal Medicine Department of Obstetrics and Gynecology, Cleveland Clinic, Cleveland, $\mathrm{OH}$; Clinical Assistant Professor, Cleveland Clinic Lerne College of Medicine of Case Western

Reserve University, Cleveland, $\mathrm{OH}$
Richard A. Krasuski, MD

Professor of Medicine and Pediatrics, Director of the Adult Congenital Heart Disease

Center, Duke University Medical Center, Durham, NC

\title{
Pregnancy in a woman with congenitally corrected transposition of the great arteries
}

\section{A 28-year-old woman \\ with congenital heart disease presents with nausea and emesis 7 weeks after her last menstrual period}

A 28-YEAR-OLD WOMAN with a history of complex congenital heart disease presents to an urgent care center with nausea and emesis, which have been worsening for 3 days. She also reports 2 weeks of constipation, with bowel movements occurring approximately every 3 days. Her most recent medical contact was 3 weeks ago for management of sinusitis that had failed to improve with azithromycin therapy. One week before the current presentation, she was prescribed trimethoprim-sulfamethoxazole for this issue. She says she has had no recent fever, lightheadedness, shortness of breath, or chest pain.

Her medical history is remarkable for congenitally corrected transposition of the great arteries (CC-TGA) with a ventricular septal defect and subpulmonic stenosis. At 5 years of age, she underwent initial surgical repair that included subpulmonic resection to relieve right ventricular outflow obstruction and closure of the ventricular septal defect to prevent chronic left-to-right shunting, a situation that could eventually lead to pulmonary vascular remodeling and pulmonary hypertension (Eisenmenger syndrome).

At age 24, she underwent implantation of a mechanical tricuspid valve to prevent the development of systemic ventricular dysfunction related to chronic, severe systemic atrioventricular valve regurgitation. Her postoperative course was complicated by complete heart block, requiring placement of a dual-chamber permanent pacemaker. Transthoracic echocardiography performed 2 months after the valve replacement

doi:10.3949/ccjm.88a.20136 demonstrated normal systemic ventricular function and no significant prosthetic valve stenosis or regurgitation. She has not undergone repeat cardiac imaging since that time.

\section{FIRST STEP IN EVALUATION}

Based on the patient's presentation, what is the most appropriate next step?

Computed tomography of the abdomen

Right heart catheterization

Urine or serum pregnancy test

Abdominal radiography

A pregnancy test is essential before a woman of childbearing age undergoes any diagnostic testing involving radiation exposure, especially if she has symptoms that could possibly be due to pregnancy. While all the above options may help with diagnosis, computed tomography, right heart catheterization, and abdominal radiography require exposure to radiation. The most appropriate and cost-effective first test to evaluate this patient's symptoms is a urine or serum pregnancy test.

\section{CASE CONTINUED:}

\section{A POSITIVE PREGNANCY TEST}

The patient says she has had no abdominal cramping, bloating, or vaginal bleeding since her last menstrual period, which was about 7 weeks before this presentation.

Her pulse is 68 beats per minute, blood pressure $112 / 70 \mathrm{~mm} \mathrm{Hg}$, oral temperature $36.8^{\circ} \mathrm{C}$ $\left(98.2^{\circ} \mathrm{F}\right)$, and respiratory rate 16 breaths per minute. 
She is alert, well-nourished, and in no acute distress. Her mucous membranes are moist. Her abdomen is without tenderness, rebound pain, guarding, or palpable masses. Auscultation of the heart reveals a crisp mechanical S1 and a grade 2 of 6 systolic ejection murmur at the left lower sternal border.

A rapid serum quantitative human chorionic gonadotropin test is positive for pregnancy.

\section{HEMODYNAMIC CHANGES OF PREGNANCY}

2 Which of the following is a normal hemodynamic adaptation of pregnancy?

Increased cardiac output

Reduced blood volume

Decreased heart rate

Increased systemic vascular resistance

Cardiac output increases during pregnancy, as the maternal cardiovascular system must undergo dynamic adaptations to meet increased metabolic demands and to support fetal growth and development through the uteroplacental circulation. Because nitric oxide is upregulated by circulating gestational hormones and the placental bed has low resistance, systemic and pulmonary vascular resistance decreases by about 30\% early in pregnancy, resulting in decreased mean arterial blood pressure. ${ }^{1}$ Beginning early in the second trimester, maternal circulating plasma volume increases by $30 \%$ to $50 \%$, peaking at the beginning of the third trimester and resulting in relative anemia and reduced blood viscosity. Heart rate also increases during that time due to increased sympathetic tone. . $^{2,3}$ Also related to sympathetic tone and increased preload, stroke volume increases by an average of $25 \%$. These physiologic changes cause cardiac output to increase steadily throughout pregnancy, peaking in the third trimester at about $50 \%$ above prepregnancy levels. ${ }^{4}$

Labor and delivery bring additional maternal hemodynamic changes that can challenge the cardiovascular system. Pain and anxiety lead to increased mean arterial blood pressure and oxygen consumption. With each uterine contraction, circulating blood volume further increases by up to $500 \mathrm{~mL}$. These rapid changes result in a further rise in maternal cardiac out-
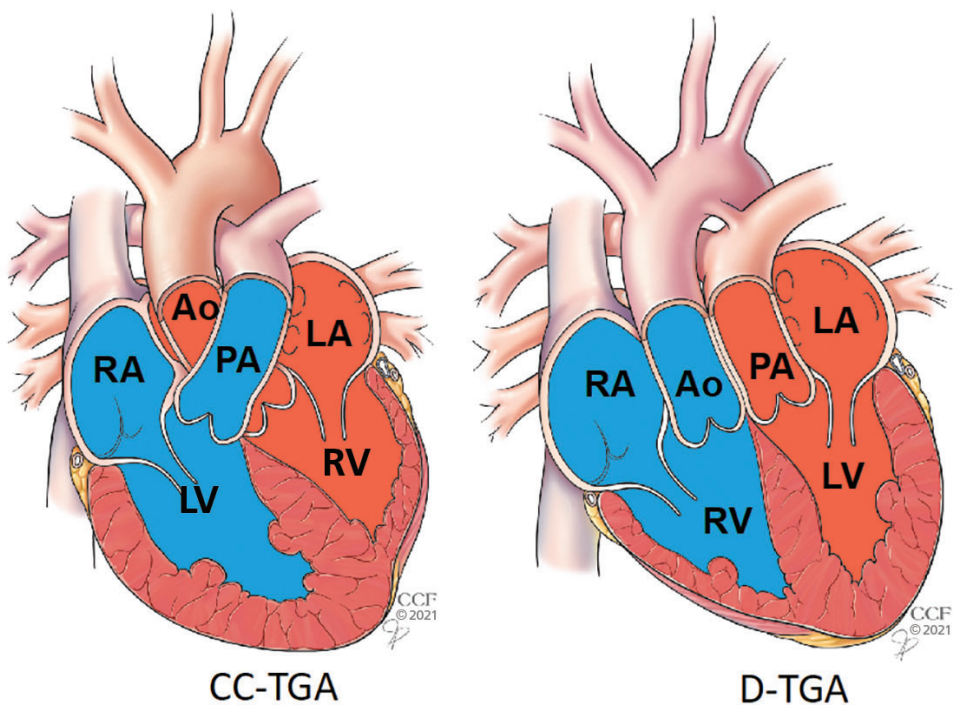

Figure 1. (Left) Congenitally corrected transposition of the great arteries (CC-TGA). The right ventricle pumps oxygenated blood in the systemic circulation, while the left ventricle pumps deoxygenated blood in the pulmonary circulation. No intervention may be needed. (Right) Dextro- or D-TGA. Pulmonary and systemic blood flows are separate, leading to rapid clinical deterioration in the first day of life unless a communication between the circulations exists or is created.

put to about $80 \%$ above prepregnancy levels, in some women reaching more than $10 \mathrm{~L} / \mathrm{min}^{5}{ }^{5}$ Some of these hemodynamic shifts can be mitigated with the use of adequate analgesia. ${ }^{6}$

After delivery, with the uterus no longer compressing the inferior vena cava, venous return increases. This, in combination with intrapartum blood shifts related to uterine contractions and a sudden increase in systemic vascular resistance following delivery of the placenta, leads to relative elevation of intracardiac filling pressures. ${ }^{6}$ Spontaneous diuresis occurs in most women over the first 24 to 48 hours after delivery. Maternal hemodynamics typically require 2 to 6 weeks to return to baseline after vaginal delivery, and usually longer after cesarean delivery.

\section{EFFECTS OF TRANSPOSITION OF THE GREAT ARTERIES}

3

Which of the following statements about TGA is correct?

In infants with dextro-transposition, a shunt is essential for survival
A pregnancy

test

is essential

before any

radiation

exposure 


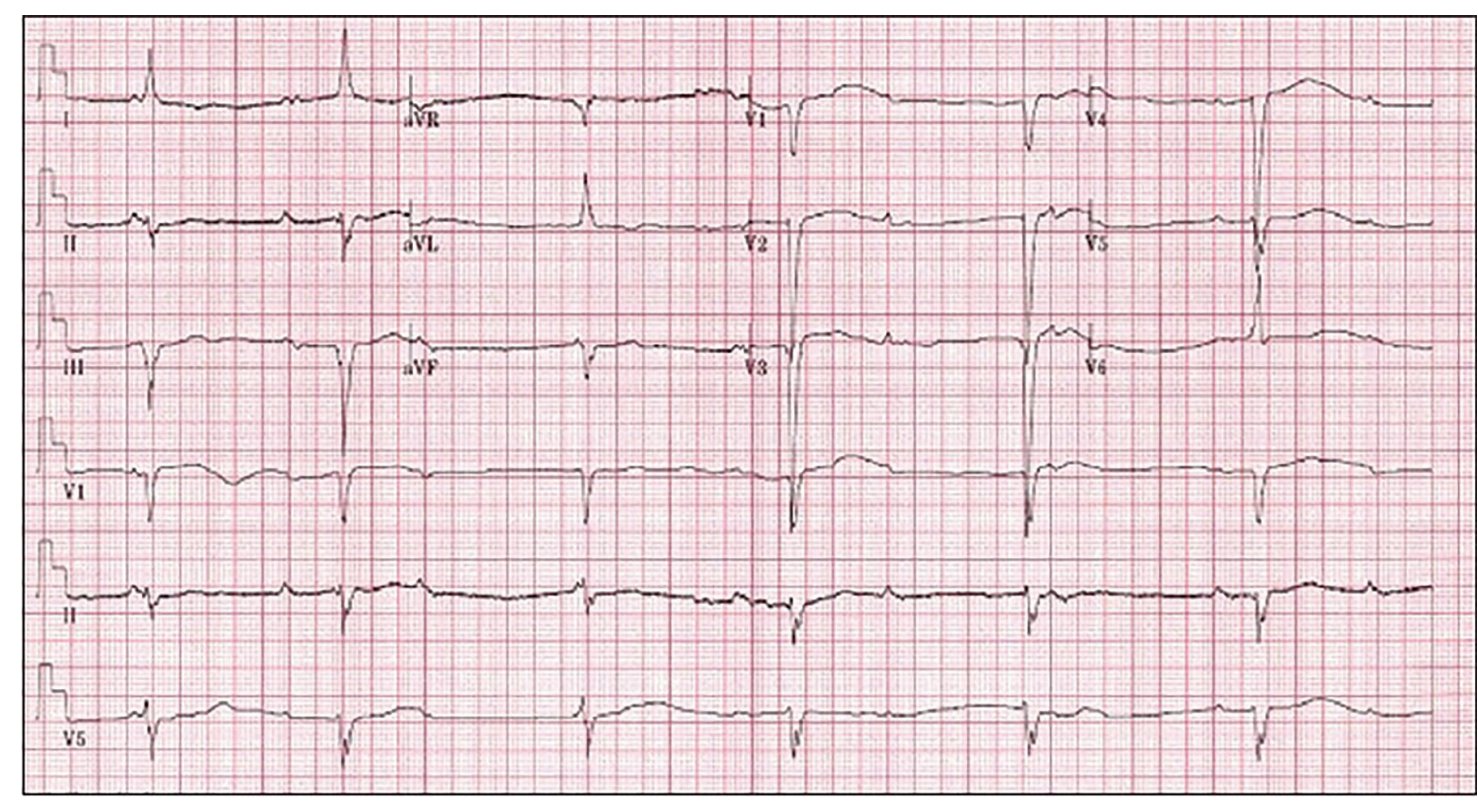

Figure 2. Electrocardiogram demonstrating complete heart block with a junctional escape rhythm in a patient with CC-TGA. The typical findings in patients with CC-TGA found here include Q waves in the inferior leads (II, III, and aVF) and right limb lead (aVR), and the absence of $Q$ waves in $V_{5}$ and $V_{6}$.

A patient with CC-TGA may develop heart failure due to dysfunction of the systemic right ventricle

Cardiac output peaks in the third trimester at about $50 \%$ above prepregnancy levels

A patient with CC-TGA may develop regurgitation of the systemic atrioventricular (morphologic tricuspid) valve patients with CC-TGA

All of the above are correct

All the above statements are correct. Heart block can occur in up to a third of

\section{In dextro-transposition,} two separate circulations

In TGA, the aorta arises from the right ventricle and the pulmonary artery from the left ventricle (Figure 1). ${ }^{7}$ In complete TGA (dextro- or D-TGA), the normal relationship between the atria and the ventricles is preserved: the right atrium empties into the right ventricle through the tricuspid valve, and the left atrium empties into the left ventricle through the mitral valve. Thus, the pulmonary circulation is separate from the systemic circulation. As a result, infants develop cyanosis and rapidly clinically deteriorate within the first day of life unless a communication between the systemic and pulmonary circulation exists through either an atrial septal defect, ventricular septal defect, patent ductus arteriosus, or atrial septostomy. ${ }^{7}$

\section{In CC-TGA, normal oxygenation}

In CC-TGA, also known as levo- or L-TGA, the right atrium empties into the left ventricle through the mitral valve and the left ventricle in turn empties into the pulmonary artery, while the left atrium empties into the right ventricle through the tricuspid valve, and the right ventricle in turn empties into the aorta.

The condition is described as "congenitally corrected" because, unlike D-TGA, the pulmonary and systemic circulations exist in series, and hence, oxygenated blood does reach the systemic circulation. In fact, the only functional difference between CCTGA and normal anatomy is that the right ventricle is responsible for pumping oxygenated blood into the systemic circulation while the left ventricle pumps deoxygenated blood into the pulmonary circulation. Hence, patients with isolated CC-TGA do not commonly require surgical or transcatheter intervention. 


\section{CC-TGA: Associated anomalies, conduction abnormalities}

CC-TGA is associated with other congenital cardiac anomalies in up to $90 \%$ of patients. The most common include deformities of the tricuspid valve, ventricular septal defects, and subpulmonic ventricular outflow obstruction. ${ }^{8}$ CC-TGA can also be associated with abnormalities of the cardiac conduction system. ${ }^{9}$

The major arrhythmogenic complication of CC-TGA is conduction system disease. Due to the presence of atrioventricular discordance, the atrioventricular node is unable to connect normally to the bundle of His. A second anteriorly positioned atrioventricular node, therefore, typically develops and gives rise to an elongated His bundle that is vulnerable to fibrosis over time. The risk of complete heart block is around $2 \%$ per year, with a cumulative lifetime incidence of 30\%. Patients with CCTGA should undergo a surveillance electrocardiogram at least yearly to screen for development of conduction system disease (Figure 2).

\section{CASE CONTINUED:}

\section{THE PATIENT CONSULTS A SPECIALIST}

Once pregnancy is confirmed, the patient is surprised, as she had been regularly using a barrier method of contraception. She is excited, as this is her first pregnancy, but becomes concerned about potential complications that she might experience due to her preexisting heart disease. She promptly schedules an appointment with her adult congenital heart disease specialist to discuss this.

\section{CARDIOVASCULAR RISK IN PREGNANCY}

Which of the following conditions pose the highest risk of pregnancy-associated maternal mortality?

Eisenmenger syndrome

Mechanical prosthetic valve

Subaortic atrioventricular valve

regurgitation with preserved subaortic

ventricular function

Moderate pulmonary valve stenosis

Eisenmenger syndrome poses the highest risk of maternal mortality of the conditions listed.

Hemodynamic changes during pregnancy, delivery, and the postpartum period may be poorly tolerated in women with congenital or acquired heart disease. Cardiovascular conditions are the leading cause of maternal mortality in the United States: $25 \%$ to $30 \%$ of pregnancyrelated deaths between 2011 and 2016 were attributable to preexisting cardiovascular disease. ${ }^{10}$

All women of childbearing age with preexisting heart disease should receive regular counseling regarding their individual risk of adverse pregnancy-related outcomes. The updated 2018 American Heart AssociationAmerican College of Cardiology guideline ${ }^{11}$ recommends that all women with congenital heart disease receive pregnancy counseling with input from a congenital heart disease specialist to determine maternal cardiac, obstetric, and fetal risks, as well as potential long-term risks to the mother (recommendation class 1 [strong recommendation], evidence level C [based on expert consensus, case studies, or standard of care]).

It is vitally important for physicians to understand the pregnancy risks associated with specific cardiac lesions and disease states. The risk for adverse pregnancy-related events in women with preexisting cardiovascular disease can be estimated based on the type and severity of the underlying cardiac disease, as well as preconception functional status.

Several clinical tools are available to assist in patient-specific preconception risk assessment. The Cardiac Disease in Pregnancy II (CARPREG II) risk score, ${ }^{12}$ which was derived from 1,938 pregnancies between 1994 and 2014, is the most contemporary. Ten predictors of adverse pregnancy-related events were identified and correlated to a weightedpoint system to assist in counseling patients:

- Previous cardiac events or arrhythmias: 3 points

- Baseline New York Heart Association class III or IV heart failure or cyanosis: 3 points

- Mechanical valve: 3 points

- Ventricular dysfunction: 2 points

- High-risk left-sided valve disease or left ventricular outflow tract obstruction: 2 points

- Pulmonary hypertension: 2 points

- Coronary artery disease: 2 points

- High-risk aortopathy: 2 points

- No prior cardiac intervention: 1 point

- Late pregnancy assessment: 1 point.

\section{Isolated CC-TGA does not commonly require surgical or transcatheter intervention}




\section{TABLE 1}

\section{World Health Organization classification of maternal cardiovascular risk}

\section{Class I}

Uncomplicated, small or mild pulmonary stenosis, patent ductus arteriosus, or mitral valve prolapse; successfully repaired simple lesions (atrial or ventricular septal defect, patent ductus arteriosus, anomalous pulmonary venous return)

Cardiovascular risk: No increased risk of maternal mortality, possible mild increase in morbidity

\section{Class II}

Unrepaired atrial or ventricular septal defect; repaired tetralogy of Fallot; supraventricular tachycardia Cardiovascular risk: Small increased risk of maternal mortality, moderate increase in morbidity

\section{Class II-III}

Mild left ventricular dysfunction; native or tissue valvular heart disease not considered World Health Organization class I or IV; Marfan syndrome without aortic dilation; aorta less than $45 \mathrm{~mm}$ in association with bicuspid aortic valve; repaired coarctation of the aorta; hypertrophic cardiomyopathy

Cardiovascular risk: As in class II or III, depending on individual

\section{Class III}

Mechanical valve; systemic right ventricle with good or mildly reduced function; Fontan circulation without complication; unrepaired cyanotic heart disease; other complex congenital heart disease; aortic dilation 40 to $45 \mathrm{~mm}$ in Marfan syndrome; aortic dilation 45 to $50 \mathrm{~mm}$ in bicuspid aortic valve disease; previous peripartum cardiomyopathy with complete recovery of ventricular function

Cardiovascular risk: Significantly increased risk of maternal mortality or severe morbidity. Expert counseling

Patients with CC-TGA should have an ECG at least yearly to screen for conduction system disease required. If pregnancy continues, intensive specialist cardiac and obstetric monitoring needed throughout pregnancy, childbirth, and for 6 weeks afterwards

\section{Class IV}

Pulmonary arterial hypertension from any cause; severe systemic ventricular dysfunction (ejection fraction $<30 \%$, New York Heart Association functional class III-IV); systemic right ventricle with moderate or severely reduced function; Fontan circulation with any complication; severe mitral stenosis; severe symptomatic aortic stenosis; Marfan syndrome with aortic dilation more than $45 \mathrm{~mm}$; aortic dilation more than $50 \mathrm{~mm}$ in bicuspid aortic valve disease; native severe coarctation of the aorta; previous peripartum cardiomyopathy without complete recovery of ventricular function

Cardiovascular risk: Pregnancy is contraindicated because of extremely high risk of maternal mortality or severe morbidity. If pregnancy occurs, discuss termination. If pregnancy continues, care as for class III

The risk of a primary cardiac event is calculated from the total points:

- $0-1$ points: $5 \%$

- 2 points: $10 \%$

- 3 points: $15 \%$

- 4 points: $22 \%$

- > 4 points: $41 \%$.

The World Health Organization has also developed a maternal cardiovascular risk classification, which is based on expert opinion
(Table 1). ${ }^{13}$

Echocardiography, cardiac magnetic resonance imaging, and exercise testing may be necessary to better define risk and understand the patient's anatomy, cardiac physiology, and functional status.

\section{Genetic counseling}

Patients with congenital heart disease or other inherited forms of preexisting cardiac disease should be offered genetic counseling. The 
prevalence of congenital heart disease is $0.8 \%$ in the general population; risk increases up to 10 -fold if congenital heart disease is also present in a first-degree relative. Rates of transmission are higher when the mother is affected compared with the father. ${ }^{14}$ In patients with autosomal dominant conditions, such as Marfan syndrome, there is a $50 \%$ chance of transmission to the fetus.

\section{COMPLICATIONS IN PREGNANCY IN WOMEN WITH CC-TGA}

5 What is the most common pregnancyassociated cardiovascular complication in women with CC-TGA?

\section{Symptomatic heart failure}

Worsening systemic atrioventricular valve regurgitation

Arrhythmia

Endocarditis

With medical management, most women with CC-TGA reach childbearing age and are healthy enough to consider pregnancy. $\mathrm{Pa}$ tients and their physicians should understand their specific risks so that they can carefully consider the appropriateness and timing of pregnancy.

Although helpful, global risk assessment tools such as CARPREG II developed to predict pregnancy-associated maternal cardiovascular risk typically do not account for the presence of a systemic right ventricle. In the maternal World Health Organization classification system, ${ }^{13}$ patients with a systemic right ventricle with normal or mildly reduced function are categorized as class III, consistent with significantly increased risk of maternal mortality or severe morbidity, and patients with moderately to severely reduced systemic right ventricular dysfunction should be advised against pregnancy (class IV).

\section{Arrhythmia risk poses challenges}

The most common pregnancy-associated cardiovascular complication seen with CC-TGA is supraventricular arrhythmia. ${ }^{15}$ It presents a management challenge, as few antiarrhythmic medications have been studied for safety and efficacy in pregnancy. Beta-blockers, especially metoprolol succinate and labetalol, are ac- ceptable in pregnancy. However, they should be used with caution in patients with CCTGA, who have an increased predisposition for heart block, which these drugs can exacerbate. In a minority of cases, supraventricular arrhythmias can lead to maternal hypoxia, which can be associated with premature delivery and low birth-weight infants. ${ }^{16}$

\section{Risk of heart failure depends on baseline function}

Systemic right ventricular dysfunction and resulting congestive heart failure symptoms are common by the third decade of life and increase in frequency as patients age. ${ }^{17}$ The World Health Organization classification of risk in patients with a systemic right ventricle is derived from the idea that the systemic right ventricle is not designed to support systemic blood flow, predisposing patients to systemic ventricular dysfunction and other cardiovascular complications of pregnancy. Although pregnancy-related changes in cardiovascular hemodynamics may provoke new-onset or worsening systemic ventricular dysfunction, most women with CC-TGA who have normal systemic ventricular function before conception do not develop symptomatic heart failure during pregnancy or postpartum. ${ }^{15,18}$

Atrioventricular valve regurgitation is typically well tolerated in pregnancy, and endocarditis is not typically a factor in patients with CC-TGA.

\section{CASE CONTINUED:} ANTICOAGULATION IN PREGNANCY

The patient engages in detailed discussions with a specialist in adult congenital heart disease and the high-risk obstetrics team about her potential risks during pregnancy. She next asks about additional considerations regarding her mechanical systemic atrioventricular valve. She currently takes warfarin $7.5 \mathrm{mg}$ daily to prevent mechanical valve thrombosis. Her international normalized ratio (INR) is within the therapeutic range.

6 How should anticoagulation be immediately handled in this patient?

Continue warfarin at her current dose of $7.5 \mathrm{mg}$ daily

Because anticoagulation can lead to fetal
Cardiac risk in pregnancy can be estimated

based on underlying cardiac disease and functional status 
complications, she should stop and remain off of all anticoagulants until after delivery

Switch to low-molecular-weight heparin during the first trimester

Mechanical heart valves are associated with elevated maternal and fetal risk, irrespective of additional structural heart disease. Thrombotic and hemorrhagic complications occur at higher rates during pregnancy in women with mechanical heart valves than in patients with congenital heart disease or bioprosthetic valves. Serious pregnancy complications occur in up to $40 \%$ of women who have a mechanical valve. ${ }^{19}$

Therapeutic systemic anticoagulation is necessary throughout pregnancy for women with a mechanical heart valve. Pregnancy is a hypercoagulable state due to increased circulating levels of fibrinogen, plasminogen activator inhibitor, and factors VII, VIII, and X. Increased platelet adhesiveness, resistance to activation of protein $\mathrm{C}$, and decreased fibrinolysis also contribute to hypercoagulability in pregnancy. ${ }^{20,21}$

\section{Warfarin and heparin both entail risk}

Warfarin, a vitamin $\mathrm{K}$ antagonist, is the most effective anticoagulant in preventing thrombotic complications in pregnant women with mechanical heart valves and is associated with lower rates of adverse maternal events compared with heparin products. However, it has traditionally been associated with fetal teratogenicity, leading to limited use during pregnancy during fetal organogenesis. Furthermore, warfarin crosses the placenta into the fetal circulation, causing systemic anticoagulation in the fetus.

Heparin products do not cross the placenta and so are considered to pose less risk to the fetus. But dosing and monitoring of heparin products during pregnancy are difficult, and heparin therapy is associated with higher rates of valve thrombosis and maternal mortality than warfarin. ${ }^{22}$

Steinberg et $\mathrm{al}^{23}$ found that women who require $5 \mathrm{mg}$ or less of warfarin daily to achieve therapeutic anticoagulation have a similar risk of adverse fetal outcomes compared with women using low-molecular-weight heparin. The current American College of Cardiol-
ogy/American Heart Association guideline on valvular heart disease management ${ }^{24}$ recommends warfarin in preference to heparin products in pregnancy if $5 \mathrm{mg}$ or less of warfarin per day is required to achieve therapeutic anticoagulation, even during the first trimester. If a higher dose is required, low-molecular-weight heparin with close monitoring of anti-Xa levels is recommended during early pregnancy. If warfarin is used, patients should be transitioned back to heparin products in the last weeks of pregnancy to minimize the risk of fetal bleeding during labor and delivery.

In addition to systemic anticoagulation, aspirin $81 \mathrm{mg}$ daily should be given to all pregnant women who have a mechanical heart valve.

\section{CASE CONTINUED: POSSIBLE DECOMPENSATION}

Because our patient requires more than $5 \mathrm{mg}$ of warfarin daily to achieve therapeutic anticoagulation, her warfarin is discontinued, and low-molecular-weight heparin is initiated and continued until the 12 th week of pregnancy, with close monitoring of anti-Xa levels. At the beginning of the second trimester, warfarin is restarted and low-molecular-weight heparin is discontinued after the patient achieves a therapeutic INR. Unfortunately, her INR proves difficult to maintain within the therapeutic range (2.5-3.5). A plan is made to resume low-molecular-weight heparin at 35 weeks of gestation in anticipation of delivery.

During a routine follow-up visit at 19 weeks of gestation, the patient reports very mild dyspnea on exertion. Her N-terminal pro-brain natriuretic peptide (NT-proBNP) level is 124 $\mathrm{pg} / \mathrm{mL}$ (reference range $<125 \mathrm{pg} / \mathrm{mL}$ ).

Transthoracic echocardiography demonstrates mild to moderate dilation of the systemic ventricle with low-normal systolic function. The subpulmonic ventricle is normal in size and function. There is moderate residual subpulmonic stenosis (peak gradient $45 \mathrm{~mm} \mathrm{Hg}$; mean gradient $28 \mathrm{~mm} \mathrm{Hg}$ ). A possible residual restrictive ventricular septal defect is also noted. Her mechanical valve is functioning normally, without evidence of regurgitation or stenosis.

As pregnancy progresses, she continues 
to experience mild dyspnea with activity. By the 34th week of pregnancy, she notes mild lower-extremity edema. She says she has no paroxysmal nocturnal dyspnea, palpitations, cough, or chest pain. Her blood pressure is $114 / 70 \mathrm{~mm} \mathrm{Hg}$. Serial echocardiograms and NT-proBNP measurements remain unchanged, despite her persistent and worsening symptoms.

7 Based on the patient's current symptoms and echocardiogram, what is the most appropriate next step in her management?

Start a diuretic to address signs of heart failure

Perform right-heart catheterization to assess heart failure

Perform transesophageal echocardiography to evaluate for prosthetic valve thrombosis No change in management at this time; continue to monitor closely

A change in management is not necessary at this time, but the patient should continue to be monitored closely.

Symptoms such as shortness of breath, orthopnea, lower-extremity edema, and sinus tachycardia may be considered normal in some women during pregnancy owing to expected hemodynamic changes. Likewise, echocardiographic chamber size, transvalvular flow velocity, and valvular regurgitation are also expected to increase as pregnancy progresses. It can be difficult to differentiate these symptoms and imaging changes from cardiac decompensation in women with heart disease. . $^{25}$

Serial NT-proBNP measurement can be helpful in differentiating whether worrisome symptoms and echocardiographic changes should be considered pathologic. On the other hand, reduced ventricular function is never considered normal during pregnancy. As such, it is important to evaluate each patient's cardiac function before determining whether a change in management strategy is necessary.

\section{CASE CONTINUED: A HEALTHY BABY}

Given her stable cardiac imaging and NTproBNP measurements, it is deemed unlikely that the patient's symptoms represent cardiac decompensation. She continues to be moni- tored closely without additional medical therapy.

As pregnancy progresses, she develops severe pelvic pain due to suspected symphysis pubis separation, as well as hemorrhoids, for which she is managed supportively.

Warfarin is continued until 35 weeks of gestation, at which time she is transitioned to low-molecular-weight heparin.

At 37 weeks, she presents with spontaneous rupture of membranes while fully anticoagulated with low-molecular-weight heparin. Despite counseling regarding bleeding risks, she opts for a primary cesarean delivery because of her concerns about the potential of worsening symptoms related to pubic symphysis separation, as she cannot be managed with neuraxial anesthesia owing to her anticoagulated state.

She undergoes primary cesarean delivery under general anesthesia. The procedure is uncomplicated and results in the delivery of a healthy infant boy with Apgar scores of 9 (of a maximum possible 10) at both 1 and 5 minutes.

Her postpartum course is uneventful. Anticoagulation with intravenous heparin is reinitiated 12 hours after delivery. Warfarin is restarted on postoperative day 3 , while continuing intravenous heparin until a therapeutic INR is achieved. She is discharged on postoperative day 7 receiving warfarin (INR 2.5) and aspirin $81 \mathrm{mg}$ daily. A nonhormonal intrauterine device is recommended for contraception.

\section{CLINICAL PEARLS}

- CC-TGA is a form of congenital heart disease characterized by ventriculoarterial and atrioventricular discordance. This makes the morphologic right ventricle responsible for pumping the systemic circulation.

- The maternal cardiovascular system must undergo tremendous hemodynamic adaptations during pregnancy, which can lead to clinical decompensation in women with cardiovascular disease.

- Risk of adverse pregnancy-related cardiovascular events in women with preexisting cardiovascular disease should be deter-

\section{A mechanical heart valve is associated with elevated maternal and fetal risk}


mined for each patient who is pregnant or considering pregnancy.

- Patients with CC-TGA can carry a pregnancy successfully without complication. The most common pregnancy-associated cardiovascular complication in patients with CC-TGA is supraventricular arrhythmia. They are also at risk for worsening systemic ventricular dysfunction and decompensated heart failure.

- Managing anticoagulation in women with a mechanical heart valve to prevent valve thrombosis during pregnancy can be difficult. When $5 \mathrm{mg}$ or less of warfarin is required to achieve therapeutic anticoagulation, it should be continued even in the first trimester. If low-molecular-weight heparin is needed, anti-Xa levels should be followed closely to ensure therapeutic anticoagulation.

Acknowledgment: The authors thank Connie S. Strouse, MD, Department of Emergency Medicine, Verde Valley Medical Center, Cottonwood, AZ, for valuable help in the early stages of preparing this manuscript.

\section{DISCLOSURES}

The authors report no relevant financial relationships which, in the context of their contributions, could be perceived as a potential conflict of interest.

\section{REFERENCES}

1. Carbillon L, Uzan M, Uzan S. Pregnancy, vascular tone, and maternal hemodynamics: a crucial adaptation. Obstet Gynecol Surv 2000; 55(9):574-581. doi:10.1097/00006254-200009000-00023

2. Ouzounian JG, Elkayam U. Physiologic changes during normal pregnancy and delivery. Cardiol Clin 2012; 30(3):317-329. doi:10.1016/j.ccl.2012.05.004

3. Sanghavi M, Rutherford JD. Cardiovascular physiology of pregnancy. Circulation 2014; 130(12):1003-1008. doi:10.1161/CIRCULATIONAHA.114.009029

4. Desai DK, Moodley J, Naidoo DP. Echocardiographic assessment of cardiovascular hemodynamics in normal pregnancy. Obstet Gynecol 2004; 104(1):20-29. doi:10.1097/01.AOG.0000128170.15161.1d

5. Abbas AE, Lester SJ, Connolly H. Pregnancy and the cardiovascular system. Int J Cardiol 2005; 98(2):179-189. doi:10.1016/j.ijcard.2003.10.028

6. Elkayam U, Goland S, Pieper PG, Silversides CK. High-risk cardiac disease in pregnancy: part II. J Am Coll Cardiol 2016; 68(5):502-516. doi:10.1016/j.jacc.2016.05.050

7. Warnes CA. Transposition of the great arteries. Circulation 2006; 114(24):2699-2709. doi:10.1161/CIRCULATIONAHA.105.592352

8. Hornung TS, Calder L. Congenitally corrected transposition of the great arteries. Heart 2010; 96(14):1154-1161. doi:10.1136/hrt.2008.150532

9. Yoon N, Kim KH, Park HW, Cho JG. Complete atrioventricular block in an adult with congenitally corrected transposition of the great arteries with interrupted inferior vena cava. J Res Med Sci 2012; 17(8):808-810. pmid:23798952

10. Centers for Disease Control and Prevention (CDC). Pregnancy mortality surveillance system. Accessed February 23, 2021. https:// www.cdc.gov/reproductivehealth/maternal-mortality/pregnancymortality-surveillance-system.htm.

11. Stout KK, Daniels CJ, Aboulhosn JA, et al. 2018 AHA/ACC guideline for the management of adults with congenital heart disease: a report of the American College of Cardiology/American Heart Association Task Force on Clinical Practice Guidelines. Circulation 2019; 139(14):e698-e800. doi:10.1161/CIR.0000000000000603

12. Silversides CK, Grewal J, Mason J, et al. Pregnancy outcomes in women with heart disease: the CARPREG II study. J Am Coll Cardiol 2018; 71(21):2419-2430. doi:10.1016/j.jacc.2018.02.076

13. Elkayam U, Goland S, Pieper PG, Silverside CK. High-risk cardiac disease in pregnancy: part I. J Am Coll Cardiol 2016; 68(4):396-410. doi:10.1016/j.jacc.2016.05.048

14. Øyen N, Poulsen G, Boyd HA, Wohlfahrt J, Jensen PK, Melbye M. Recurrence of congenital heart defects in families. Circulation 2009;
120(4):295-301. doi:10.1161/CIRCULATIONAHA.109.857987

15. Kowalik E, Klisiewicz A, Biernacka EK, Hoffman P. Pregnancy and long-term cardiovascular outcomes in women with congenitally corrected transposition of the great arteries. Int J Gynaecol Obstet 2014; 125(2):154-157. doi:10.1016/j.ijgo.2013.10.022

16. Therrien J, Barnes I, Somerville J. Outcome of pregnancy in patients with congenitally corrected transposition of the great arteries. Am J Cardiol 1999; 84(7):820-824. doi:10.1016/s0002-9149(99)00444-0

17. Fredriksen PM, Chen A, Veldtman G, Hechter S, Therrien J, Webb G. Exercise capacity in adult patients with congenitally corrected transposition of the great arteries. Heart 2001; 85(2):191-195. doi:10.1136/heart.85.2.191

18. Connolly HM, Grogan M, Warnes CA. Pregnancy among women with congenitally corrected transposition of great arteries. J Am Coll Cardiol 1999; 33(6):1692-1695. doi:10.1016/s0735-1097(99)00046-7

19. van Hagen IM, Roos-Hesselink JW, Ruys TP, et al. Pregnancy in women with a mechanical heart valve: data of the European Society of Cardiology Registry of Pregnancy and Cardiac Disease (ROPAC). Circulation 2015; 132(2):132-142. doi:10.1161/CIRCULATIONAHA.115.015242

20. Bremme KA. Haemostatic changes in pregnancy. Best Pract Res Clin Haematol 2003; 16(2):153-168. doi:10.1016/s1521-6926(03)00021-5

21. Kujovich JL. Hormones and pregnancy: thromboembolic risks for women. Br J Haematol 2004; 126(4):443-454. doi:10.1111/j.1365-2141.2004.05041.x

22. James AH, Brancazio LR, Gehrig TR, Wang A, Ortel TL. Low-molecular-weight heparin for thromboprophylaxis in pregnant women with mechanical heart valves. J Matern Fetal Neonatal Med 2006; 19(9):543-549. doi:10.1080/14767050600886666

23. Steinberg ZL, Dominguez-Islas CP, Otto CM, Stout KK, Krieger EV. Maternal and fetal outcomes of anticoagulation in pregnant women with mechanical heart valves. J Am Coll Cardiol 2017; 69(22):2681-2691. doi:10.1016/j.jacc.2017.03.605

24. Nishimura RA, Otto CM, Bonow RO, et al. 2014 AHA/ACC guideline for the management of patients with valvular heart disease: a report of the American College of Cardiology/American Heart Association Task Force on Practice Guidelines. J Am Coll Cardiol 2014; 63(22):e57-e185. doi:10.1016/j.jacc.2014.02.536

25. Regitz-Zagrosek V, Roos-Hesselink JW, Bauersachs J, et al. 2018 ESC guidelines for the management of cardiovascular diseases during pregnancy. Kardiol Pol 2019; 77(3):245-326. doi:10.5603/KP.2019.0049

Address: Richard A. Krasuski, MD, Division of Cardiology, Duke University Medical Center, DUMC 3010, Durham, NC 27710;

richard.krasuski@duke.edu 